\section{A cryoSEM Method for Preservation and Visualization of Calcified Shark Cartilage (And Other Stubborn Heterogeneous Skeletal Tissues)}

Mason N. Dean ${ }^{1}$, Stanislav N. Gorb ${ }^{2}$ \& Adam P. Summers ${ }^{1}$ Ecology \& Evolutionary Biology, University of California Irvine, Irvine, CA USA

${ }^{2}$ Evolutionary Biomaterials Group, Max Planck Institute for Metals Research, Stuttgart, Germany mdean@uci.edu

Biological materials interest biologists and engineers for their complex interactions among constituents and unique mechanical properties. While the heterogeneous tissue structure and its material properties are responsible for intriguing biomechanics, they pose challenges for sectioning, particularly in regions with stark tissue boundaries. Microscopists level this playing field when sectioning samples by embedding them in paraffin or plastic; but for scanning electron microscopy, natural morphology must be preserved without sacrificing the sample's surface contours. Here we outline a simple preparation method for visualizing in cryoSEM the calcified cartilage of sharks and rays (elasmobranch fishes), a layered biocomposite that has traditionally been considered difficult to prepare for microscopy.

Elasmobranch skeletons are made almost entirely of a cartilage similar to the gel-like hyaline cartilage in our joints. Yet in most parts of the body, the cartilage is covered by a superficial bark of abutting tiles (tesserae) that tessellate the surface but lie beneath the fibrous perichondrium that wraps each skeletal element (Figs.1,2A) (Applegate,1967; Clement,1992; Dean \& Summers, 2006; Dingerkus et al., 1991, Kemp \& Westrin,1979).

The structure is a multilayer composite comprised of a ceramic mineralized layer overlain by fibrous tissue and underlain by a viscoelastic gel (Dean et al., 2005; Dean \& Summers, 2006). Interactions between the layers are complex: the fibrous tissue grades into its insertion in tesserae, adjoining adjacent tiles and anchoring them to the perichondrium and underlying unmineralized cartilage (Dean \& Summers, 2006; Summers, 2000). The mineralized and unmineralized cartilage phases are also tightly related, apparent from the ubiquity of chondrocytes (cartilage cells), scattered through both the unmineralized matrix and tesserae, which are rife
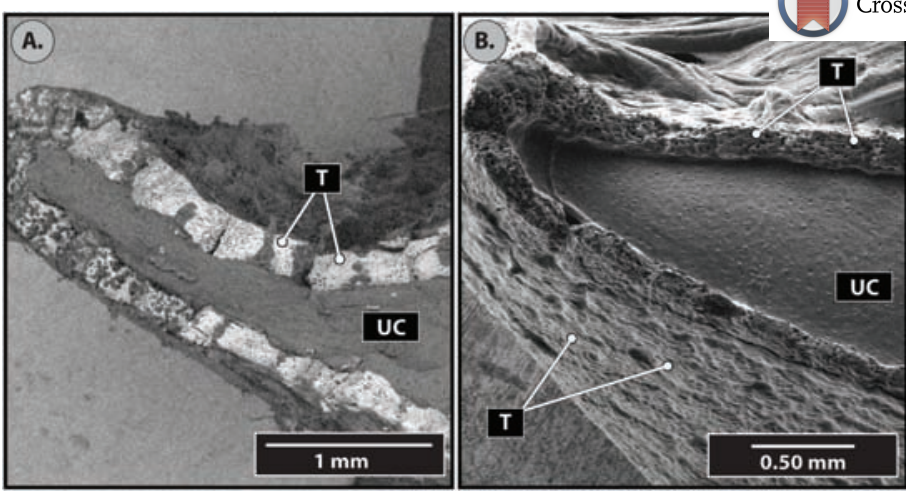

Figure 1. Anatomy of the tessellated skeleton and comparison between two tissue preparation methods. In cross-section, the cartilage of sharks and rays appears as a core of uncalcified cartilage (UC) tiled with a surface bark of mineralized tesserae ( $T$ ) overlain by fibrous perichondrium. Specimens of stingray jaw prepared by critical point drying (panel $A$, shown in a backscatter electron micrograph where calcified tissue is white) are greatly distorted by collapse of the uncalcified tissue, whereas those prepared by the cryoSEM method discussed in the current article (panel B) provide a more accurate window into the tissue's native state. Note the camera view in panel B is somewhat oblique and tesserae can be seen in cross-section at the top of the image and in surface view, covered by perichondrium, at the bottom.

with mineralized lacunae (Fig. 2) (Kemp \& Westrin, 1979; Dean \& Summers, 2006; Summers, 2000).

Despite the involvement of the two cartilage phases our EDX and indentation assays show the material boundary is sharp; moving from the uncalcified cartilage into the tesserae, calcium and phosphate levels immediately skyrocket and material stiffness modulus increases by 3 orders of magnitude to roughly $2 \mathrm{GPa}$ (Dean et al., 2005; Dean \& Summers, 2007; Dean, 2007). Like other soft-hard musculoskeletal boundaries (e.g., tendon entheses in bone), the transition region represents an integrated biocomposite. The whole may be more than the parts: as in straw and mud combining to form a thatch that is strong and tough, in tessellated cartilage we believe that commingling of the hydroxyapatite ceramic with the viscoelastic gel results in a skeleton that is both stiff and has a high damping capacity.

As we hypothesize that the combination of the two tissue phases results in the intriguing fatigue-resistant properties of the tessellated skeleton, in our SEM work we are most interested in the morphological interaction of the tissue phases. It is this interaction that makes sample preparation so awkward: methods that are perfect for one
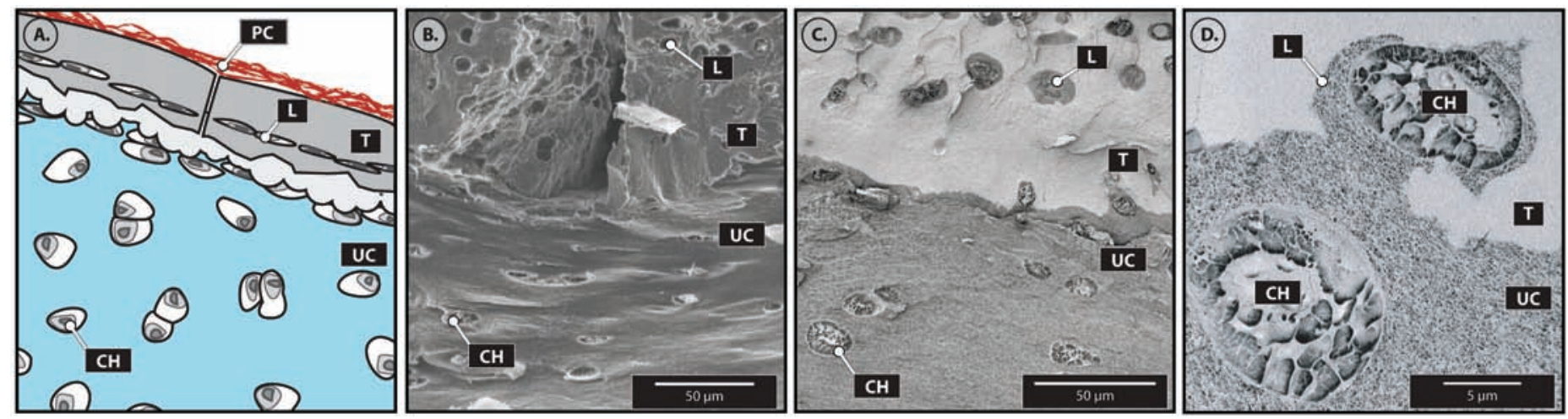

Figure 2. Fine scale morphology of the interfacial region of stingray cartilage. The schematic (panel A) illustrates the basic "sandwich" morphology of tessellated cartilage, in which tesserae (T) surmount uncalcified cartilage (UC) and in turn are covered by perichondrium (PC). A comparison of preservation methods illustrates that critical point drying (panel B) results in collapse and folding of the uncalcified tissue relative to our cryoSEM method (panels $C$, $D$ ) where chondrocytes $(\mathrm{CH})$ remain ovoid, filling their lacunae $(L)$ in both tissue phases. Our method also ensures a planar surface across the tissue interface (panel D), maintaining the fine scale interactions of chondrocytes and tesserae. 


\section{Electron/Ion Beam Instrument Engineer \\ University of Oregon CAMCOR}

The University of Oregon's Center for Advanced Materials Characterization in Oregon (CAMCOR) is seeking applications for a full time staff position to begin in the Fall of 2008. A strong background in maintaining, trouble shooting and upgrading electron/ ion/x-ray beam instruments, and associated high voltage, vacuum, mechanical, and electrical systems is required.

This position will be located in the new Lorey Lokey Integrated Science Laboratory, a state of the art nano and micro science analytical instrument facility designed specifically for exceptional nano-science performance. It will house the latest electron, ion and $\mathrm{x}$-ray beam instrumentation available including a Zeiss Ultra TFEM, FEI Quanta 200 E-SEM, Cameca SX50 and SX100 microprobes, Ion-TOF SIMS, Phi Auger Spectrometer, FEI Titan TEM, FEI Helios Dual Beam FIB, Thermo XPS and various assorted coaters, etchers, and other vacuum deposition systems.

The successful candidate will have a BS in a beam microscopy related field (or equivalent experience) and an extensive background in instrument field service with major instrument manufacturers. Significant practical experience is required involving troubleshooting high vacuum electron and ion beam instrumentation at both the system, component, and PC board levels.

They also must be able to read and understand schematics for electronic circuits and systems and have experience in embedded systems hardware design and programming in assembler and C. Title (e.g. instrument engineer or research specialist) and Rank (e.g. research assistant) for the position will be assigned based on the successful candidate's qualifications.

The successful applicant will be involved in modifying/improving instrumentation capabilities to enable the equipment to support more fully our unique research needs and will be expected to work intimately with the scientific staff and research faculty. We seek candidates with a demonstrated commitment to working effectively with students, faculty, and staff from diverse backgrounds.

Interested persons should send a resume with a detailed description of work experience and skills, and arrange for two letters of recommendation to be sent to: CAMCOR Instrument Engineer Search Committee, 1253 University of Oregon, Eugene, OR, 97403-1253. To be assured of full consideration, application materials must be received by November 20, 2008. For further information, contact John Donovan (donovan@ uoregon.edu). University of Oregon is an AA/EEO employer committed to cultural diversity.

\section{The New Dual Standard.}

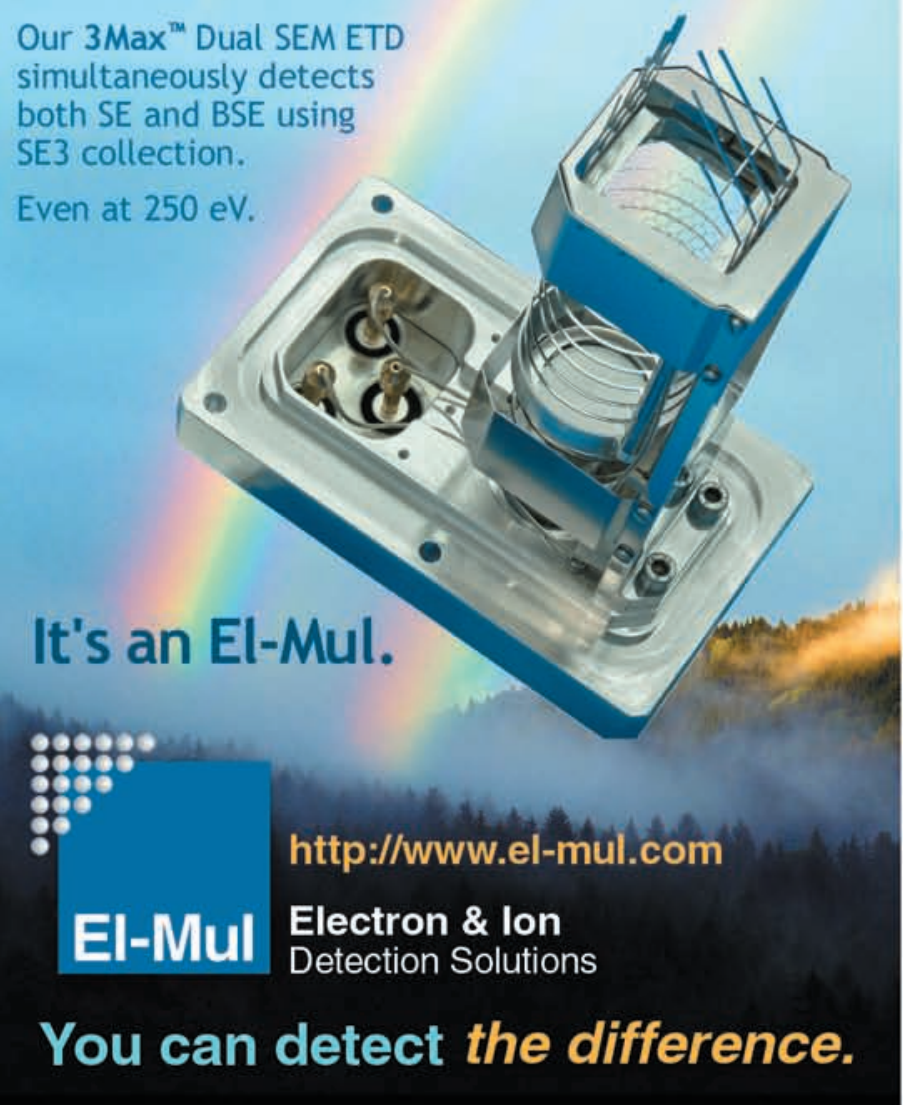

INDUSTRY RESEARCH

MEDICINE

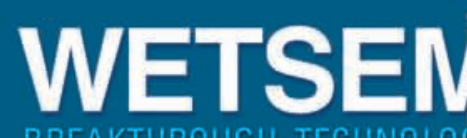

High Resolution Imaging

of Fully Hydrated Samples

Greatly Reduces Prep Time

Capsule Fits Standard SEMs

Safe and Simple to Use

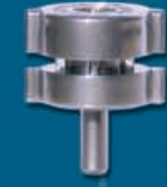

QX-102
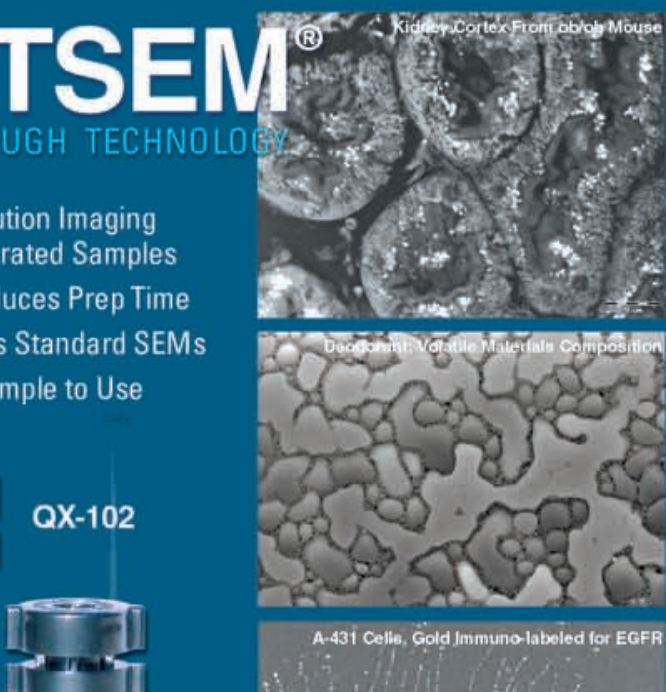

A-43i Cella, Gold Immuno-labeled lor EGFR
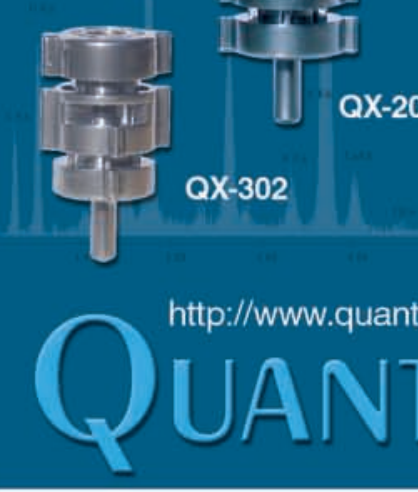

QX-302

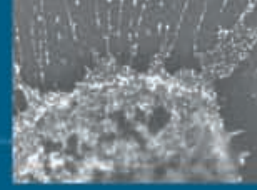

http://www.quantomix.com
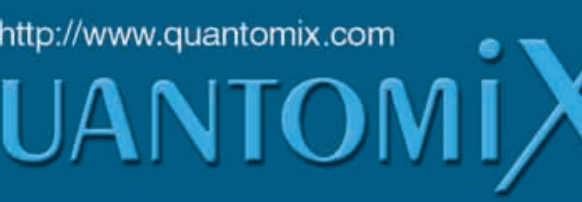

(R) 


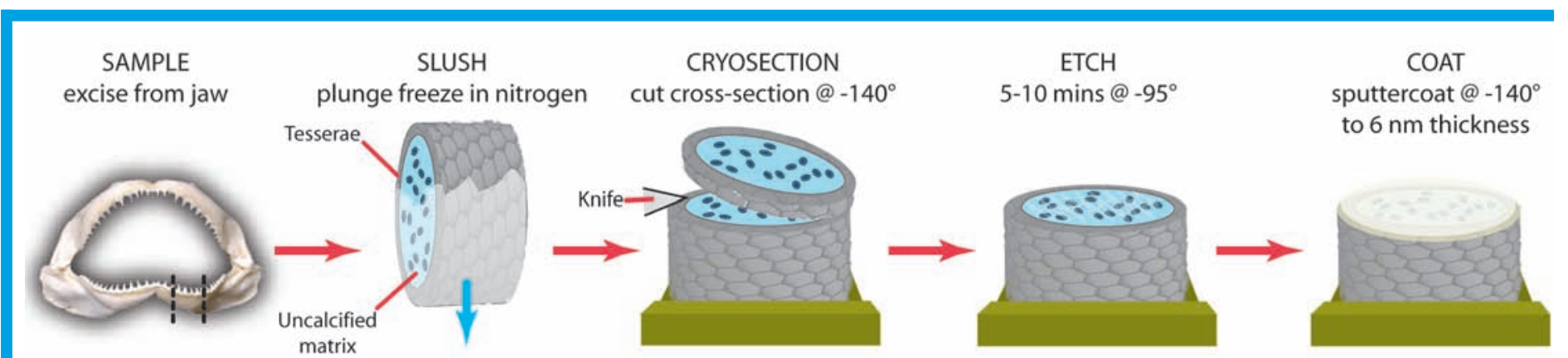

Figure 3. Diagram of the described cryoSEM methodology for sectioning and visualizing elasmobranch cartilage.

tissue may ignore or be detrimental to the morphology of another. For example, previous studies have focused mostly on the tesserae themselves at the expense of the uncalcified matrix, in some cases, examining air-dried specimens or employing bleach protocols to remove the perichondrium (e.g. Applegate, 1967; Kemp \& Westrin, 1979; Dingerkus et al., 1991; Clement,1992). Decalcification (e.g. in EDTA) facilitates sectioning for light microscopy (e.g. Kemp \& Westrin,1979; Clement,1992; Eames et al., 2007) but it is unclear if this alters morphologies native to the calcified phase. Critical point drying for SEM provides useful views of tesserae but distorts the organic phase, resulting in drastic changes in sample geometry from the shriveling of unmineralized cartilage and perichondrial and inter-tesseral fibrous interactions (Fig.1 A, 2B). In addition, because tesserae are stiff with complex interdigitations, cross-sectioning elasmobranch skeletal samples for traditional SEM is damaging to the micromorphology of interest. Synchrotron radiation can provide ultra-high resolution tomograms of hydrated samples that preserve intertesseral joint morphologies; however, radio-transparent soft tissues are not visible.

Low temperature scanning electron microscopy (cryoSEM) obviates many of these issues; here, we discuss a cryoSEM methodology that provides a rapid and effective means of visualizing tessellated cartilage (Fig. 3). Previously we have used a cryoSEM approach, in order to visualize tiny secretory droplets in the contact between fly attachment devices (pulvilli) and smooth surfaces (Gorb, 2006). Here we report on the cryoSEM protocol for examining an ontogenetic series of fresh stingray (Urobatis halleri) jaws (ranging from $1-5 \mathrm{~cm}$ in the longest dimension and $0.5-1 \mathrm{~cm}$ thick), however, we imagine this protocol to be useful for many skeletal tissues with sharp material boundaries.

First, we removed samples from the muscular and tendinous tissue, leaving a skeletal element wrapped primarily in perichondrium. We then clamped the specimen tightly in a metal holder and plunge-froze the stub in slushed liquid nitrogen before introducing it to the $-140^{\circ}$ cooled preparation chamber (Gatan Alto 2500) (Fig. 3 ). In our microscope (Hitachi-4800), this chamber is equipped with a cold scalpel blade with a long, user-controlled handle for sample sectioning; we used the scalpel to "pop" the top off of the sample. This created a new surface parallel to the holder, with the low temperatures ensuring a fracture plane much cleaner than that obtained in room temperature sectioning. This is the step most prone to user-error; success is largely dependent on the sharpness of the scalpel and the tightness of the cryoholder's purchase on the sample.

We then increased specimen temperature to $-95^{\circ}$ and etched the sample for 10-15 minutes to remove the thin layer of water or/ and contamination occasionally caused by ice crystals that obscure surface morphology. The preparation chamber was returned to its original temperature $\left(-140^{\circ}\right)$; longer etching periods typically resulted in visible distortion of the uncalcified matrix. After transferring the sample to the SEM chamber, the tissue could be visualized with the greatest detail; however, to avoid charging (e.g. Fig. 2B), we typically sputter coated it with gold palladium (layer thickness $-6 \mathrm{~nm}$ ) and then visualized the sample at low accelerating voltage $(1-2 \mathrm{kV})$ at the sample stage temperature of $-120^{\circ}$. The strength of this simple protocol is its ability to stabilize the tissue interface and provide a smooth-faced cross-section of the skeletal element (Fig. 2). Not only is the tidemark between cartilage phases rendered as a planar surface (Fig. 2C), but chondrocytes and their pericellular tissues are clearly visible throughout (Figs. 2D, 4) and fibrous tissue insertions into mineralized tissues are maintained in their natural configurations. From samples prepared this way, we were able to learn ultrastructural details of native tessellated cartilage and quantify aspect ratios and densities for chondrocytes and tesserae from the same images (Dean, 2007; Dean \& Summers, 2007).

Although the technique works well for visualizing soft tissue phases interacting intimately with hard tissue phases, it has shortcomings for more ambitious and higher resolution applications. Whereas the basic interfacial morphology is maintained, there are surely soft tissue distortions resulting from the phase changes of water during freezing (Hunziker et al., 1984, 1997; Tavakol et al., 1993). Although we are confident that the densities and aspect ratios of chondrocytes are accurate, the webbing effect visible in the uncalcified extra-cellular matrix is likely a freezing artifact rather than a representation of the collagen fiber organization of the tissue (Figs. 3, 4) (Hunziker et al, 1984, 1997).

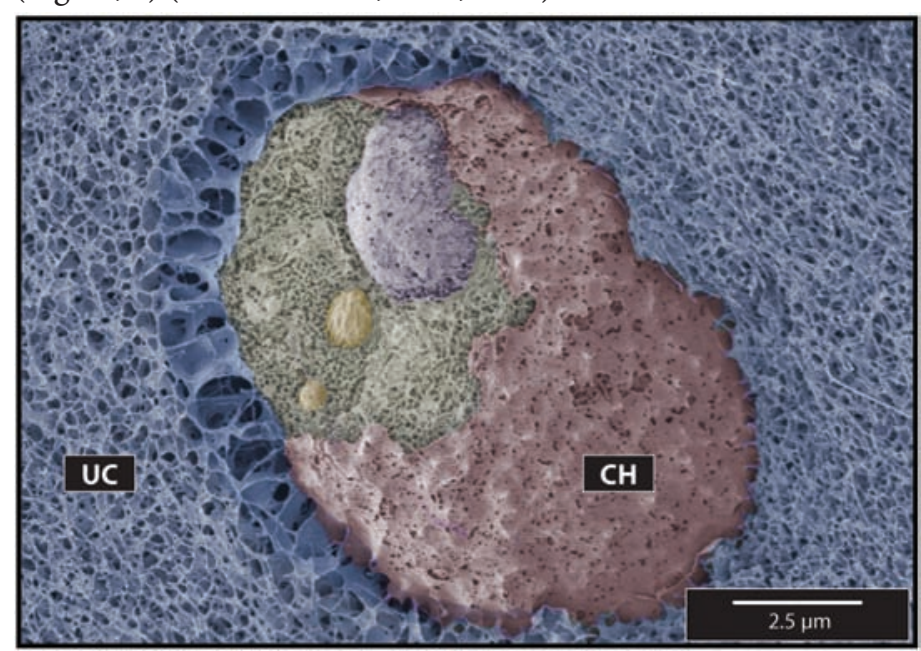

Figure 4. Pseudocolored image of a cow articular cartilage chondrocyte (CH) prepared using the described method. The webbed appearance of the surrounding uncalcified cartilage (UC) matrix is likely a preservation artifact. Organelles are visible and well-preserved within the cell; this was more common for mammalian than stingray tissue and may point to structural differences between the two cartilages. 
High-pressure freezing (HPF) would more accurately preserve the matrix but has maximum size constraints that, given the structure of this skeletal material, would necessitate tissue samples that are too small for our questions of interest (Studer et al. 1995). Tesserae are relatively macroscopic structures ranging from several hundred microns to nearly 1 millimeter across; in most dried jaws of large sharks they can be seen with the naked eye. To visualize the tissue interface using HPF preparation method, the requisite sample dimensions and the stiffness of the tesserae would demand a very sharp and precise tissue punch. In addition, as efficacy of HPF on cartilage is strongly time-dependent, tissue would need to be prepared immediately following the death of the fish; this would make field collection of specimens very difficult, although HPF of tessellated cartilage may be possible for laboratory specimens.

Although our methodology was designed to look at a specific material, we imagine the order of events will be useful for other skeletal tissues with soft/hard interfaces. However, durations of different stages of the protocol will naturally have to be varied according to specimen size and the results may vary across cartilage types. We found that cow cartilage, sectioned similarly, yielded much greater detail at the cellular level, such that organelles were clearly visible in fractured chondrocytes (Fig. 4). This visible difference may point to compositional differences between mammalian and elasmobranch cartilage and warrants some further investigation.

This research was supported by a Journal of Experimental Biology Traveling Fellowship to M.N.D. and a National Science Foundation grant to M.N.D. and A.P.S. (IOB-0616322).

\section{References}

Applegate, S. P. (1967). A survey of shark hard parts. In Sharks, Skates and Rays, eds. P. W. Gilbert R. F. Mathewson and D. P. Rall, pp. 37-66. Maryland: Johns Hopkins Press.

Clement, J. G. (1992). Re-examination of the fine structure of endoskeletal mineralization in Chondricthyes: implications for growth, ageing and calcium homeostasis. Australian Journal of Marine and Freshwater Research 43, 157-181.

Dean, M.N. and A.P. Summers, (2007). Ontogeny, morphology and mechanics of the tessellated skeleton of cartilaginous fishes. Integrative and Comparative Biology. 47: e27.

Dean, M.N. (2007) Ontogeny, morphology and mechanics of the tessellated skeleton of cartilaginous fishes. Journal of Morphology. 268(1066).

Dean, M. N., Chiou, W.-A. and Summers, A. P. (2005). Morphology and ultrastructure of prismatic cartilage calcification. Microscopy and Microanalysis 11,1196-1197.

Dean, M. N. and Summers, A. P. (2006). Cartilage in the skeleton of cartilaginous fishes. Zoology 109,164-168.

Dingerkus, G., Seret, B. and Guilbert, E. (1991). Multiple prismatic calcium phosphate layers in the jaws of present-day sharks (Chondrichthyes; Selachii). Experientia 47, 38-40.

Eames, B.F., Allen, N., Young, J., Kaplan, A., Helms, J.A. \& Schneider, R.A. (2007) Skeletogenesis in the swell shark Cephaloscyllium ventriosum. Journal of Anatomy 210: 542-554.

Gorb, S.N. (2006). Fly microdroplets viewed big: a Cryo-SEM approach. Microscopy Today 14(5): 38-39

Hunziker, E. B., Herrmann, W., Schenk, R. K., Mueller, M. and Moor, H. (1984). Cartilage ultrastructure after high-pressure freezing, freeze substitution, and low-temperature embedding .1. Chondrocyte ultrastructure - implications for the theories of mineralization and vascular invasion. Journal of Cell Biology 98, 267-276.

Hunziker, E. B., Michel, M. and Studer, D. (1997). Ultrastructure of adult human articular cartilage matrix after cryotechnical processing. Micros. Research and Technique 37, 271-284.

Kemp, N. E. and Westrin, S. K. (1979). Ultrastructure of calcified cartilage in the endoskeletal tesserae of sharks. Journal of Morphology 160, 75-102.

Studer D., Michel M., Wohlwend M., Hunziker E.B. and Buschmann M.D. (1995). Vitrification of articular cartilage by high-pressure freezing. Journal of Microscopy. 179(3):321-32.

Summers, A. P. (2000). Stiffening the stingray skeleton - An investigation of durophagy in myliobatid stingrays (Chondrichthyes, Batoidea, Myliobatidae). Journal of Morphology 243, 113-126.

Tavakol K., Miller R.G., Bazett-Jones D.P., Hwang W.S., McGann L.E. and Schachar N.S. (1993) Ultrastructural changes of articular cartilage chondrocytes associated with freeze- thawing. Journal of Orthopedic Research. 11(1): 1-9.

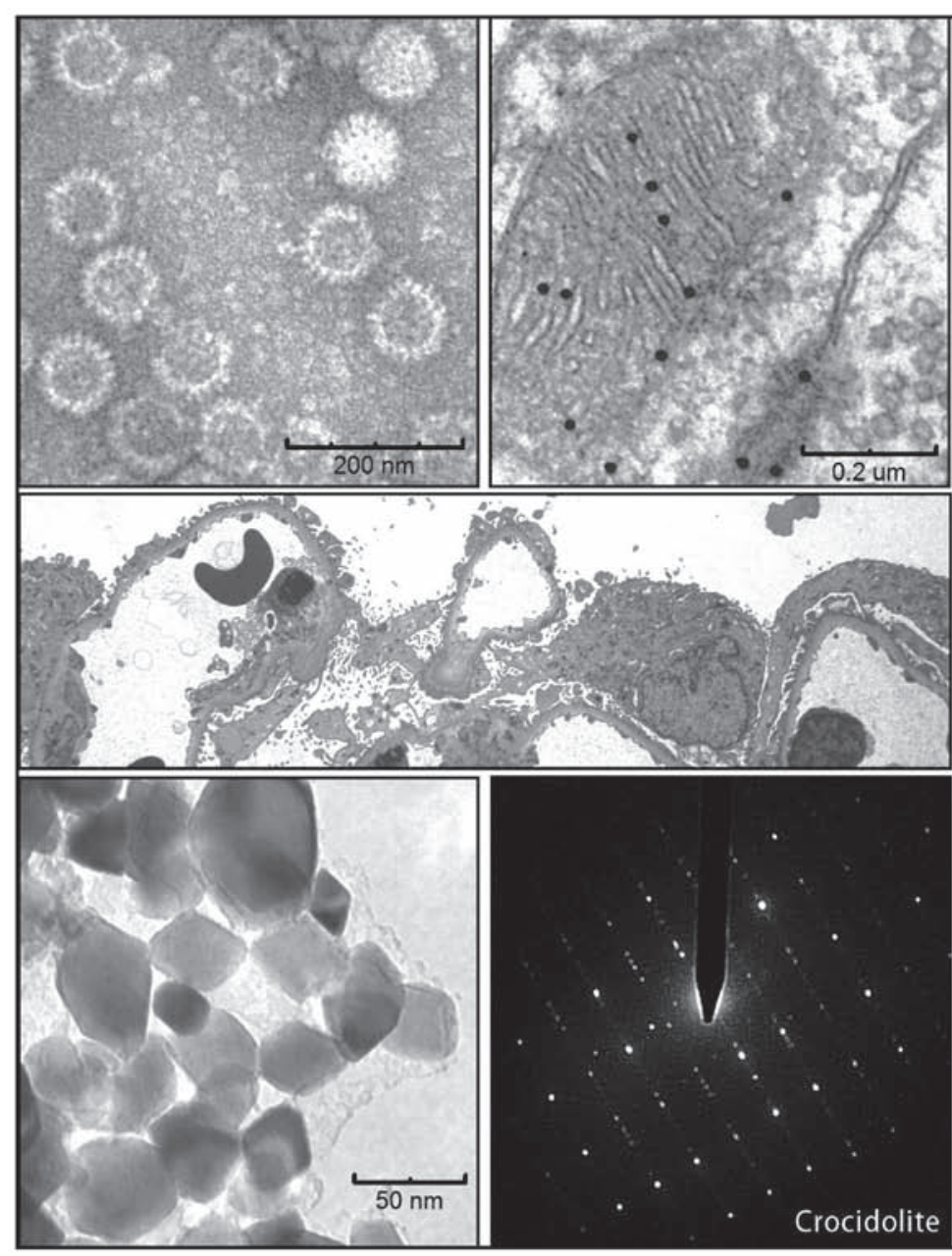

Affordable TEM

camera systems for

research, healthcare,

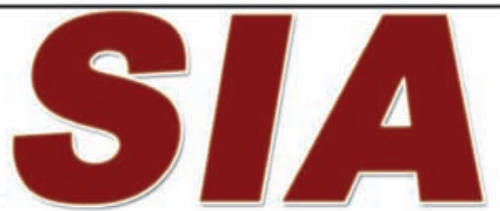

education, and industry

since 2001

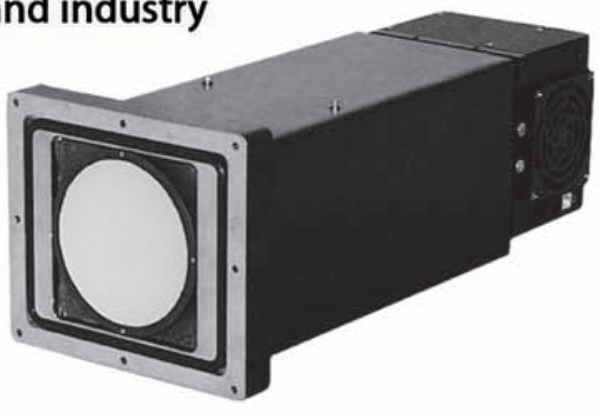

1 to 16 Megapixels, slow scan and TV

Magnification factor of 1 on bottom mounted cameras

Diffraction beam stop on side mounted cameras

Reliable, easy to use and upgrade

Standard and custom configurations for any TEM

Compatible with existing TEM accessories

Scientific Instruments and Applications

2773 Heath Lane; Duluth, GA; 30096

(770) 232 7785; www.sia-cam.com

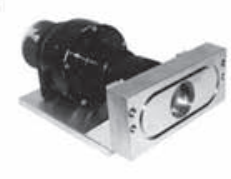

